\title{
Research on Construction of the University Sports Network Information Platform (USNIP)
}

\author{
Yongjie Liang \\ Department of Sports, Guizhou University of Finance and Economics, Guiyang 550025, China
}

gzcd2012@163.com

\begin{abstract}
Keywords: university sports network information platform, sports curriculum education, sports education networking.
\end{abstract}

\begin{abstract}
Based on internet and China education network, point at the characteristics and shortcomings of university sports education, take network as the carrier, the establishment of college sports network information platform covering the function of student users health information collection, health information guidance, sports theory teaching, sports theory assessment, both makes up for the regret that the proportion of theoretical knowledge in college physical education is insufficient, and meet the need that modern university students eager to learn targeted sports theoretical knowledge to guide classroom practice, to achieve the reform purpose of college sports curriculum system cultivating students' comprehensive quality and competence oriented. This paper gives detailed design process and implementation approaches about how to build a university sports network information platform, has some practical significance and application value, which plays an active role for each university sports network information platform establishment and improvement, also plays an important role for college students physical health research. In addition, for university physical education teachers, establishment and operation of this system, they can easily complete the theoretical aspect education, with differentiated targeted teaching to improve the quality of education.
\end{abstract}

\section{Introduction}

In many universities, teaching resources are shortage, cannot provide a lot of classrooms at the same time to take the unify sport theory course, this contradiction has been an old problem in university physical education. With the popularization of computer, the development and maturity of internet-based distance education, building a sports theory course network information system is one of the effective ways to solve this contradiction. China internet network information center released the 26th China internet development statistics report in 15 July 2010. The report showed that as of June 2010, Chinese netizens reached 420 million, mobile phone users 277 million, internet penetration rate rose to $31.8 \%$. This set of latest data shows that China's information ministry after years of building information network hardware, Chinese internet CNNET has already deep into the lives of 1.3 billion people, has become the main source for ordinary people to access, to publish information. Education network CERNET (China education and research network) is a national academic computer network, a national investment construction, the Ministry of education responsible for the management, Tsinghua and other universities undertake the construction and operation management. It is mainly targeted at education and research institutions, is the national largest nonprofit internet network, in 1996 was recognized by the State Council as one of the four backbone networks, universities, schools and other educational and research institutes connecting CERNET are more than 1000, in which colleges and universities are more than 800 . From the above two points, we can conclude that: compared to 10 years ago the basis of internet-based distance education undoubtedly has a qualitative leap, construction and coverage area of basic education network has covered all domestic universities. Teachers of domestic higher education institutions, undergraduates, graduate students, doctoral students and researchers have become accustomed to learn and carry out research work under the environment of education network or internet. The 
widely popularization of internet, education network ensure the hardware infrastructure of internet-based university physical education system construction.

\section{The Necessity of Constructing USNIP}

Since 1999 China launched the distance education pilot, there are 68 network universities have developed internet higher diploma education, domestic financial, bonds, accounting and other industries take a lot of digital, networking, real-time education and assessment system, save a lot of human resources and costs for the respective agencies. College physical education, include sports professional theoretical knowledge, has always been simply pass on to students only in the time of practice class, the amount and integrity of knowledge cannot be guaranteed. The students are often scanty for the benefits of the learned sports items to their physical, psychological, interpersonal, etc., or there is doubt without answer. Therefore, the construction of a university sports network information platform, point at the course characteristics of college sports, with remote physical theory of education and learning progress assessment function, is necessary, also is adapt to the trend of modern information technology education.

\section{The Overall Plan of USNIP}

(1) HTTPS protocol.Based on consideration of network security, to prevent the data invasion and destruction, college sports education system must use the HTTPS protocol. HTTPS (full name: Hypertext Transfer Protocol over Secure Socket Layer), is the HTTP channel targeted at safe, simply speaking is the secure version of HTTP [1]. Namely, add SSL layer into HTTP, the security infrastructure of HTTPS is SSL, therefore the encrypt details need SSL. It is an URI scheme (abstract identifier system), is used for secure HTTP data transmission[2]. HTTPS: URL indicates that it uses HTTP, but in HTTPS exist a default port and an encryption / authentication layer different from HTTP (between HTTP and TCP). The services provided by SSL protocol mainly include: (1) authenticate the user and the server, make sure to send data to the correct client and server; (2) encrypt data to prevent data being stolen in the way; (3) maintain the integrity of the data, ensure that the data is not changed during transmission.

(2) The system architecture.This system uses B/S (Browser/Server architecture) three layer structure model, composed by web browser (Browser) and server (including Web application server and database server) [3]. In this structure, the user's working interface is realized by the WWW browser, very small part of the business logic is realized on the front side (Browser), and the main business logic is realized on the server side (Server), server side accesses to the database. The biggest advantage of using such a structure is that it can guarantee centralized
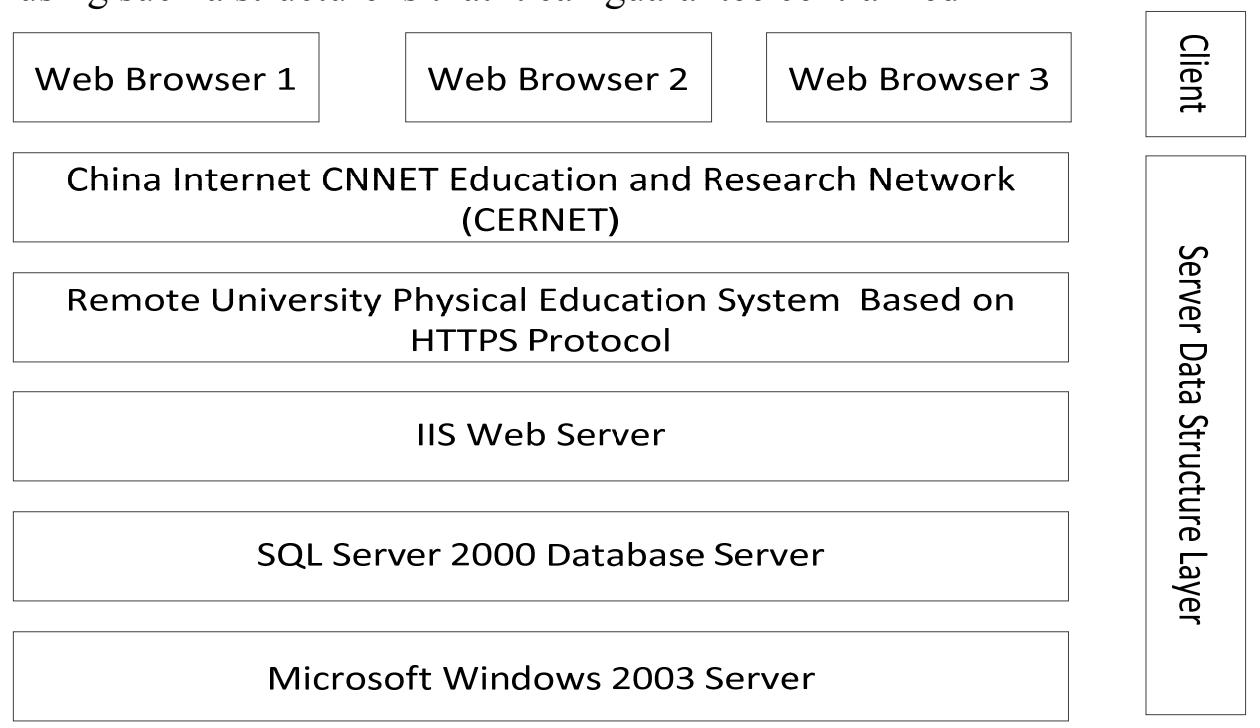

Fig. 1 USNIP Architecture 
processing services, program databases publishing information in the system are finished focus on the server, the client after installing the operation systems and browsers can work, greatly simplify the client hardware requirement and configuration process, the physical structure is shown in Figure 1: USNIP architecture.

The system structure is divided into six layers, from bottom to top, the first layer: operating system layer, using Microsoft's Windows server2003 as the network operating system or server operating system, Windows server 2003 can provide high performance, high reliability and high security; the second layer: database system layer, SQL Server 2000 can take full advantage of Windows NT's strengths, support local and remote system management and configuration, students basic information, test database classification, and the score pooled analysis require the support of SQL Server 2000 database; the third layer: IIS Web server, as the database interface layer, through IIS Web application server collect data information into each interface system; the fourth layer: application service layer, university sports network information platform uses ASP.NET and HTTPS protocols technology to exchange information with database server, at the same time through China internet (CNNET) or China education and research network (CERNET) to exchange information with the front side browse; the fifth layer: interactive network layer, China internet (CNNET), China education and research network (CERNET); the sixth layer: user layer client, oriented to terminal users (teachers and students), provide them with operation interface, the users through a web browser (Browser) can conduct information exchange with server data structure layer [4].

(3) The functional structure of USNIP.From the functional point of view, USNIP is divided into user management module; network curriculum management module; test database maintenance management module; real exam module; marking audit analysis module; system analysis management module; user's health information collection analysis module; system functional structure is shown in Figure 2.

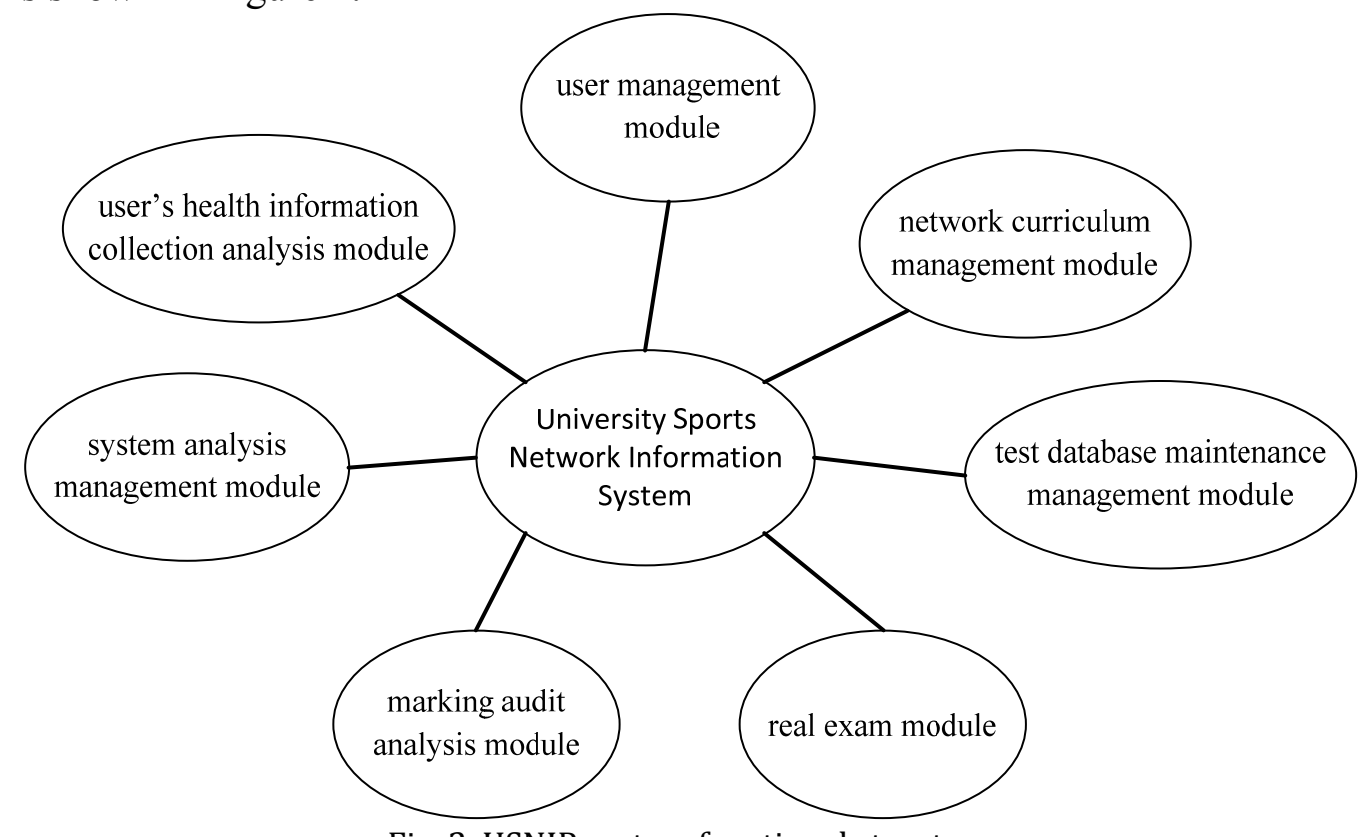

Fig. 2 USNIP system functional structure

(1) User management.Users of this system are divided into three categories: system administrators, teachers and students. On the permission classification, the system administrator has the highest permission, teachers manage the progress of students' theory course, select question bank and other related data, students have the basic permission, for belong to what classroom teachers, learn according to the progress divided by teachers, examine theoretical knowledge and other basic management [5].

(2) Network curriculum management. Network curriculum is an important form of distance education, with respect to traditional media, has information dynamic update and instant interactive and other features. First, the formulation and management of network curriculum schedule, in full accordance with the schedule of students' instructors, according to the progress of classroom practice, 
corresponding increase some knowledge for students to learn. Second, use the teaching units composed of multimedia elements, organize and complete network curriculum content by hypertext, flash animations. Third, interest knowledge points link redirect. The main carrier of university sports Network information platform is web, knowledge points are realized through hyperlinks. Course topics should focus on interesting and ornamental, to inspire students' initiative in the learning process[6].Fourth, teaching interaction. University sports network information platform management system through real-time dialogue or message function to complete interactive teaching based on teachers subsystem and students subsystem, make the system is also equipped the important role of realizing preaching, tuition, disabusing in traditional teaching.

(3) Question database maintenance management. Question database maintenance management module is the foundation of examination system, realize the establishment and maintenance of examination question database, and include questions addition, modify, insert, store, delete, print and other functions.

(4) Real-time test administration. Teachers according to the learning phase activate the test exercises of each stage, the system randomly generates a relate knowledge node examination paper based on examination question database, and then students complete the answer sheet operation, after the end of the examination, submit papers.

(5) Marking audit. Open examination scoring system supports automatically marking examination paper and evaluation functions. The system makes full use of the characteristics of the computer automatically process, take the manner of automatic scoring for objective questions, after candidates submit the answer result, the system automatically mark according to the standard answer, the result is available after the end of examination.

(6) System management. Include user management, rights management and questions management, the main function is user and question management, and control each teacher and student to use, manage permission. Specifically divided into four sub-modules, namely teacher management module, student management module, examination question database management module, rights management module.

(7) User's health information collection and analysis. User's health information include student user's basic physiological data, ,exercise habits, hobbies, physique test data, psychological test data, and other data, after completing collecting the above data, can directly use college sports network information platform to analyze and sort, the obtained conclusion can provide support for teacher user's research.

\section{References}

[1] KONG Jun, YI Qing, Research on the user-oriented sports information integration service platform construction [J], Wuhan Institute of Physical Education Journal, 2009, 43 (8): 55-58.

[2] KONG Jun, YI Qing, University sports management information system based on C / S and B / S [J], Wuhan Institute of Physical Education Journal, 2007, 41 (10): 14-17.

[3] CHEN Chuanming, ZHENG Xiaoyao, The designation and implementation of basic computer paperless examination system [J], Computer Era, 2010, 41 (7): 67-69.

[4] ZHANG Weijiang, The designation and implementation of title English paperless examination system [J], Software Guide, 2010 (6): 60-62.

[5] SUN Xiaojie, The designation and implementation of open examination system [J], Tianjin Polytechnic University Journal, 2010 (6): 85-88.

[6] CUI Kai, Design and implementation of network test system [J], Science Volkswagen, 2010 (6): 104. 